\title{
Persistent Presence of the Anti-Myelin Oligodendrocyte Glycoprotein Autoantibody in a Pediatric Case of Acute Disseminated Encephalomyelitis Followed by Optic Neuritis
}

Akihiko Miyauchi $^{1}$ Yukifumi Monden ${ }^{1}$ Meri Watanabe ${ }^{2}$ Hideo Sugie $^{1}$ Mitsuya Morita ${ }^{3}$ Takeshi Kezuka ${ }^{4}$ Mariko Momoi ${ }^{1}$ Takanori Yamagata ${ }^{1}$

${ }^{1}$ Department of Pediatrics, Jichi Medical University, Shimotsuke, Tochigi, Japan

2 Department of Ophthalmology, Jichi Medical University, Shimotsuke,

Address for correspondence Yukifumi Monden, MD, Department of Tochigi, Japan

${ }^{3}$ Department of Neurology, Jichi Medical University, Shimotsuke,

Tochigi, Japan

${ }^{4}$ Department of Ophthalmology, Tokyo Medical University, Shinjyuku,

Tokyo, Japan Pediatrics, Jichi Medical University, 3311-1 Shimotsuke, Tochigi 3290498, Japan (e-mail: mon4441977319@jichi.ac.jp).

Neuropediatrics 2014;45:e2.

ERRATUM
The publisher regrets an error in the sequence of author names in the above article of Neuropediatrics, published online on March 7, 2014 (DOI: 10.1055/s-0034-1371179). The correct sequence of the author names is Akihiko Miyauchi, Yukifumi Monden, Meri Watanabe, Hideo Sugie, Mitsuya Morita, Takeshi Kezuka, Mariko Momoi, and Takanori Yamagata as appears above. The author Akihiko Miyauchi is thereby correctly listed as the first author of the above article. 